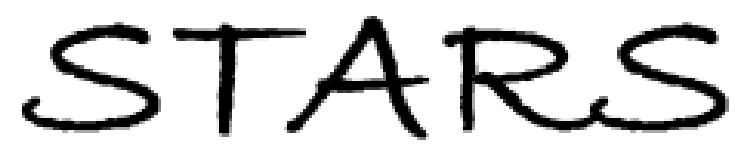

University of Central Florida

STARS

$1-1-1996$

\title{
Multisoliton Generation By Laser Modes In A Frequency Doubling Medium
}

Pascal Agin

George I. Stegeman

University of Central Florida

Find similar works at: https://stars.library.ucf.edu/facultybib1990

University of Central Florida Libraries http://library.ucf.edu

This Article is brought to you for free and open access by the Faculty Bibliography at STARS. It has been accepted for inclusion in Faculty Bibliography 1990s by an authorized administrator of STARS. For more information, please contact STARS@ucf.edu.

\section{Recommended Citation}

Agin, Pascal and Stegeman, George I., "Multisoliton Generation By Laser Modes In A Frequency Doubling Medium" (1996). Faculty Bibliography 1990s. 1532.

https://stars.library.ucf.edu/facultybib1990/1532

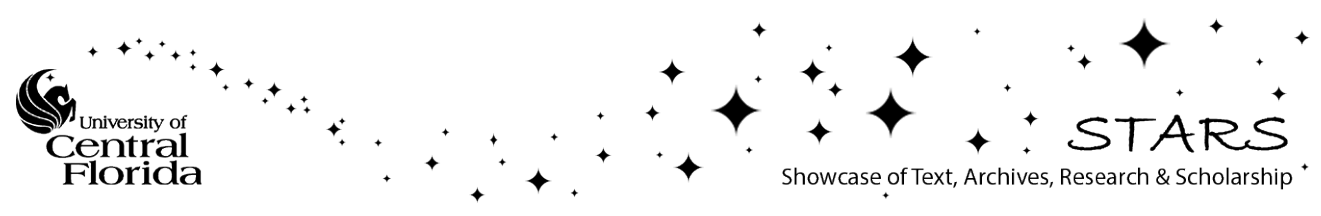




\section{Multisoliton generation by laser modes in a frequency doubling medium}

Cite as: Appl. Phys. Lett. 69, 3996 (1996); https://doi.org/10.1063/1.117849

Submitted: 22 August 1996 . Accepted: 21 October 1996 . Published Online: 19 August 1998

Pascal Agin, and George I. Stegeman
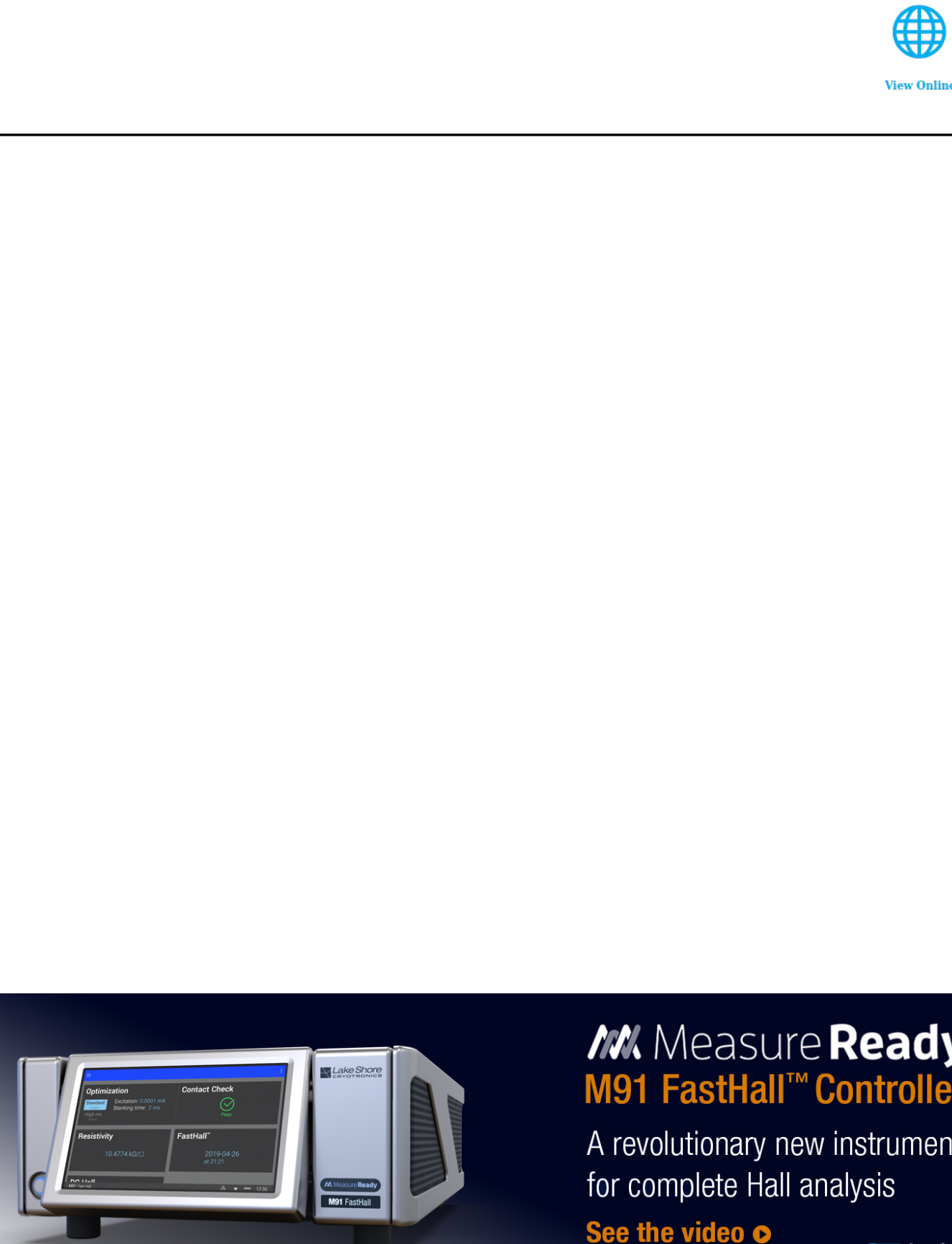

\section{mo Measure Ready} M91 FastHall ${ }^{\text {TM }}$ Controller

A revolutionary new instrument for complete Hall analysis

\section{See the video 0}

LakeShore 


\title{
Multisoliton generation by laser modes in a frequency doubling medium
}

\author{
Pascal Agin \\ Ecole Polytechnique, 91128 Palaiseau cedex, France
}

George I. Stegeman a)

Center for Research and Education in Optics and Lasers and Department of Physics, University of Central Florida, Orlando, Florida 32826

(Received 22 August 1996; accepted for publication 21 October 1996)

We demonstrate that the number of solitons generated by laser modes with multiple power maxima
in a phase-matchable, second-order, nonlinear optical medium can be explained by a model based
on field overlap and neighboring soliton interactions. (c) 1996 American Institute of Physics. [S0003-6951(96)02352-2]

Filimentation of laser beams in bulk materials at high input powers has been interpreted as the self-trapping of field maxima via the competition between self-focusing (via third order nonlinearities) and beam spreading (via diffraction). ${ }^{1}$ Stable filaments called spatial solitons propagate without diffraction for special material conditions. In the mid 1970's, it was predicted that 2D (two transverse beam dimensions) self-trapped beams could also be formed via the competition between diffraction and second order nonlinearities during second harmonic generation (SHG). ${ }^{2}$ 2D quadratic solitons were observed in 1995 near the phase-matching condition for Type II SHG in (KTP). ${ }^{3}$ These experiments utilized a $\mathrm{TEM}_{00}$ laser mode and only one spatial soliton was generated. ${ }^{3}$ However, Type II SHG allows the flexibility of not only using higher order laser modes $\mathrm{TEM}_{m n}$ with $(m+1) \times(n+1)$ field maxima, but also SHG via the mixing of different spatial modes, one for each polarization. Here we show that spatially well-defined 1D and 2D patterns of solitons can be generated in this way, and that the details of the initial field overlap as well as the interaction between neighboring solitons determine the number of solitons obtained.

The governing equations for the three-wave interaction in a Type II configuration at phase matching are ${ }^{3}$

$$
\begin{aligned}
& \frac{\partial A_{1}}{\partial z}+\frac{1}{2 i k_{1}}\left[\frac{\partial^{2} A_{1}}{\partial^{2} x}+\frac{\partial^{2} A_{1}}{\partial^{2} y}\right]=i \Gamma A_{2}^{*} A_{3}, \\
& \frac{\partial A_{2}}{\partial z}-\rho_{\omega} \frac{\partial A_{2}}{\partial x}+\frac{1}{2 i k_{2}}\left[\frac{\partial^{2} A_{2}}{\partial^{2} x}+\frac{\partial^{2} A_{2}}{\partial^{2} y}\right]=i \Gamma A_{1}^{*} A_{3}, \\
& \frac{\partial A_{3}}{\partial z}-\rho_{2 \omega} \frac{\partial A_{3}}{\partial x}+\frac{1}{2 i k_{3}}\left[\frac{\partial^{2} A_{3}}{\partial^{2} x}+\frac{\partial^{2} A_{3}}{\partial^{2} y}\right]=2 i \Gamma A_{1} A_{2},
\end{aligned}
$$

where $z$ and $(x, y)$ are respectively the propagation and transverse beam directions, $k_{i}$ are the wavevectors, $A_{1}$ and $A_{2}$ are the envelopes of the two, orthogonally polarized, fundamental fields and $A_{3}$ is the envelope of the secondharmonic field, $\rho_{\omega}$ and $\rho_{2 \omega}$ are respectively the walk-off angles of the extraordinary fundamental and secondharmonic, and $\Gamma$ is the nonlinear coupling coefficient. For KTP at $\lambda=1064 \mathrm{~nm}, \Gamma=6 \mathrm{~cm}^{-1}$ for an input intensity of 1 $\mathrm{GW} / \mathrm{cm}^{2}$, and $\rho_{\omega}=0.19^{\circ}$ and $\rho_{2 \omega}=0.28^{\circ}$. The equations were integrated numerically using a split-step approach with

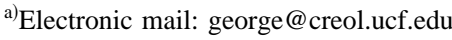

a grid of $256 \times 256$ points and a 15 -point mesh absorber. Without the absorber, the energy was conserved to better than $0.1 \%$, indicating that the propagation algorithm used was adequate.

We numerically launched two fundamental $\mathrm{TEM}_{m n}$ laser modes defined by

$$
\begin{aligned}
A(x, y)= & \sqrt{\frac{2 P}{\pi \omega_{0}^{2}}} c_{m} c_{n} H_{m}\left(x \sqrt{\frac{2}{\omega_{0}}}\right) H_{n}\left(y \sqrt{\frac{2}{\omega_{0}}}\right) \\
& \times \exp \left[-\frac{x^{2}+y^{2}}{w_{0}^{2}}\right],
\end{aligned}
$$

where $\omega_{0}$ is the beam waist, $P$ is the power of the beam, $I_{0}=2 P / \pi \omega_{0}^{2}$ is the peak intensity of the $\mathrm{TEM}_{00}$ laser mode, $c_{m}=\left(2^{m} m !\right)^{-1 / 2}$ is a normalization coefficient and $H_{m}(X)$ is the $m$ th order Hermite polynomial. We used $\omega_{0}=20 \mu \mathrm{m}$ at the input and assumed equal input power for the two orthogonal fundamental polarizations. We defined an operational soliton locking threshold as the input intensity at which the ordinary polarized beam waist after five diffraction lengths is smaller than the input beam waist.

Simulations were performed for a number of laser mode combinations. For some cases, the results were exactly what would be expected intuitively based on simple field overlap. For example, for two identical laser modes $\mathrm{TEM}_{m n}$, the maximum number of solitons was simply $(m+1) \times(n+1)$. For example for $\mathrm{TEM}_{10}$, the two, well-defined, overlapped maxima led to the generation of two solitons, symmetric about the $x-z$ plane.

Here we discuss the most interesting and not intuitively obvious results which occur for two distinctly different laser modes. Consider an ordinary $\mathrm{TEM}_{10}$ and an extraordinary $\mathrm{TEM}_{00}$ fundamental mode without walk off. For $I_{0}<2$ $\mathrm{GW} / \mathrm{cm}^{2}$, the parametric gain length $L_{n l} \gg L_{d}$ (diffraction length) and two beams are observed for both the ordinary fundamental as well as the second harmonic, and only one beam for the extraordinary fundamental. The behavior is essentially linear. When $I_{0}$ was increased, four distinct fundamental beams are observed and self-focusing begins to dominate diffraction for the two middle beams. As $I_{0}>8.5$ $\mathrm{GW} / \mathrm{cm}^{2}$, two solitons are formed from the innermost beams, each consisting of a second harmonic ( $\mathrm{SH}$ ) component and the two fundamentals, as seen in Fig. 1(a). The two outer 
(a)

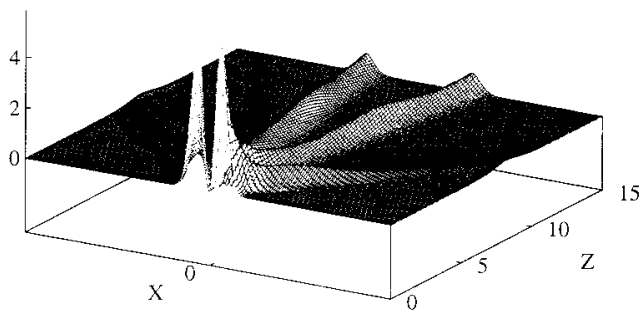

(b)

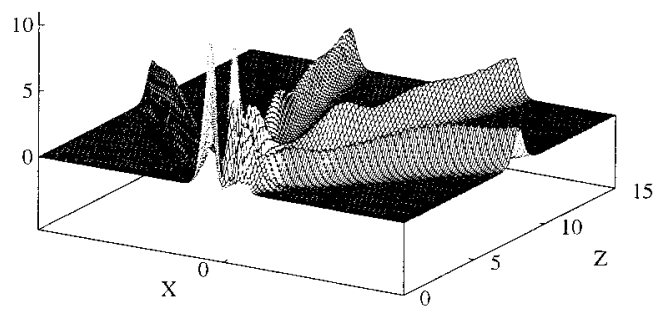

(c)

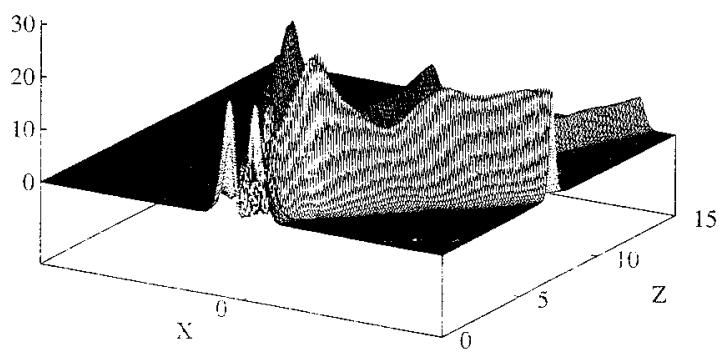

FIG. 1. A slice in the $x-z$ plane of the evolution of the ordinary polarized, fundamental intensity (input as $\mathrm{TEM}_{10}$ ) with an extraordinary $\mathrm{TEM}_{00}$ incident field. $I_{0}=10 \mathrm{GW} / \mathrm{cm}^{2}$ for (a) and $15 \mathrm{GW} / \mathrm{cm}^{2}$ for (b), all without walk off, $z$ is given in diffraction lengths. In (c), walk off is included and $I_{0}=30 \mathrm{GW} / \mathrm{cm}^{2}$.

beams continue to diffract on propagation. Finally, for $I_{0}>14 \mathrm{GW} / \mathrm{cm}^{2}$, four solitons are generated, as seen in Fig. $1(\mathrm{~b})$.

The intuitively unexpected generation of four solitons at high intensities can be explained by a simple model. Four distinct "zones" can be defined in the simple end-on view of both fundamental input beams in Fig. 2(a). The detailed evolution of all three interacting beams over the first diffraction length is shown in Fig. 3 for $I_{0}=10 \mathrm{GW} / \mathrm{cm}^{2}$. Near the input,

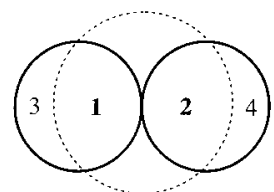

(a)

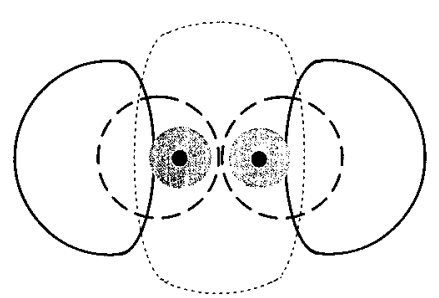

(b)
FIG. 2. Simple end-on views of the ordinary fundamental $\mathrm{TEM}_{10}$ (solid line), the extraordinary fundamental $\mathrm{TEM}_{00}$ (dotted line), and the secondharmonic (dashed line) (a) at the input. (b) after a propagation distance of less than one diffraction length. The second-harmonic down conversion occurs mainly near its maximum intensity (black points) and generates two additional beams (gray area) for the ordinary fundamental on down conversion.
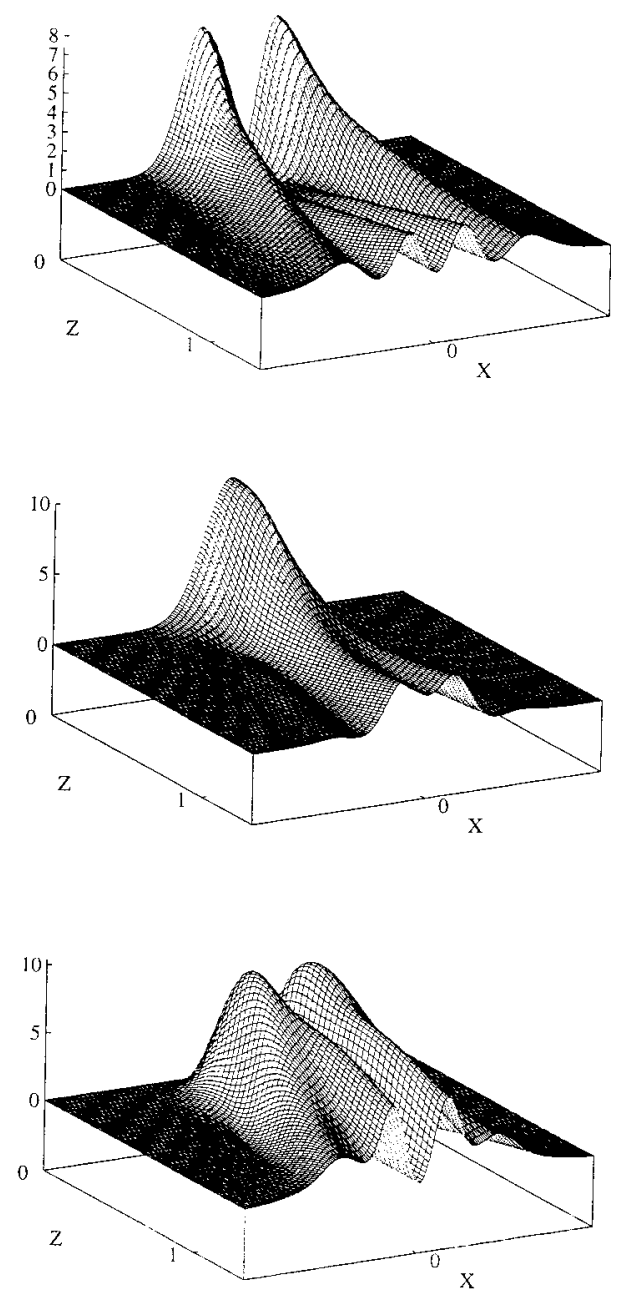

(c)

FIG. 3. Detailed evolution of the ordinary fundamental [(a), TEM 10 at the input], the extraordinary fundamental [(b), $\mathrm{TEM}_{00}$ at the input], and the second harmonic (c) over one diffraction length for an input intensity of 10 $\mathrm{GW} / \mathrm{cm}^{2}$.

the strongest up conversion to $\mathrm{SH}$ occurs in regions 1 and 2 as shown in Fig. 3(c), i.e., the solid dark regions in Fig. 2(b). Inspection of Fig. 3 indicates that the $\mathrm{SH}$ peaks, where the overlap between the two fundamental modes is maximized, is closer to the beams' center than the original maxima in the ordinary fundamental. Because of the preferential depletion of the ordinary fundamental on the beam center side of its inputted maximum and some diffraction on propagation, its original maxima are shifted further from the beam center than they were right at the input. This results in a separation between the $\mathrm{SH}$ and ordinary fundamental maxima. After a short propagation distance, less than $L_{d}$ at high $I_{0}$, strong down conversion of the second harmonic into the ordinary fundamental occurs where the SH optimizes its overlap with the extraordinary fundamental. Thus two new maxima are generated in the ordinary fundamental at high enough $I_{0}$, located closer to the beam center than the remaining (after depletion) normal mode peaks for a total of 4 maxima at around one $L_{d}$ (or less) from the input. Because the ordinary fundamental beam depletion is large, the peak intensity of the down-converted waves can be larger than what remains of the original undepleted ordinary fundamental, as seen in Fig. 3(a). The larger $I_{0}$, the more dominant these inner peaks 


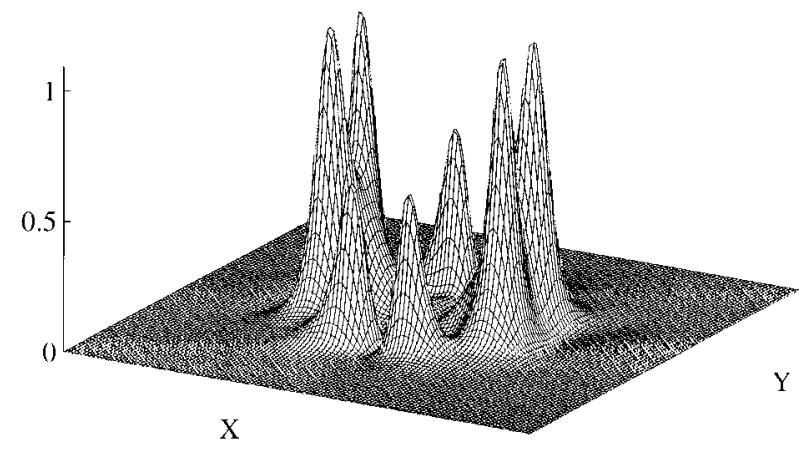

FIG. 4. The output intensity of the ordinary polarized fundamental (input as $\left.\mathrm{TEM}_{10}\right)$ in the $x-y$ plane after 4 diffraction lengths. The extraordinary mode was $\mathrm{TEM}_{01} \cdot I_{0}=20 \mathrm{GW} / \mathrm{cm}^{2}$.

can be. This four-fold spatial modulation is transferred to both the harmonic and extraordinary fundamental through successive up- and down-conversion processes. Depending on $L_{d} / L_{n l}\left(\propto I_{0}\right), 0,2$, or 4 spatial solitons can be formed. Note that the threshold intensity for the generation of the two solitons from regions 3 and 4 is larger than for the two inner beams from regions 1 and 2 . Although the paths of the four soliton maxima diverge along the line on which the field maxima lie, the net transverse soliton momentum in the $x$ $y$ plane is conserved because the local field maxima each have an equal but opposite $k$-vector spectrum and no net transverse momentum is introduced by the inputs. Because the beams formed in zones 3 and 4 are further from the center of the ordinary fundamental beam, they emerge with a higher transverse momentum due to diffraction.

Walk-off breaks the beam overlap symmetry along the $x$ axis and each of the four solitons generated has a different soliton-locking threshold intensity. In fact, because the extraordinary fundamental now propagates to the right, the maximum intensity of both fundamentals in zones 2 and 4 is larger than in zones 1 and 3 as compared to the no walk-off case. Thus, four solitons are generated successively with increasing $I_{0}$ for the beams coming from zones $2,4,1$, and 3, respectively, as seen in Fig. 1(c).

A good test of the model is SHG via the TEM 10 and $\mathrm{TEM}_{01}$ fundamental modes. There are four inner and four outer zones, and in general 8 beams are possible. And, as observed in Fig. 4, 8 solitons can indeed be generated. Note that such processes can be used with high order modes to produce 1D and 2D spatial patterns.

These simple rules can break down for higher order modes. Solitons due to closely spaced adjacent maxima merge over certain intensity ranges, reducing the number of

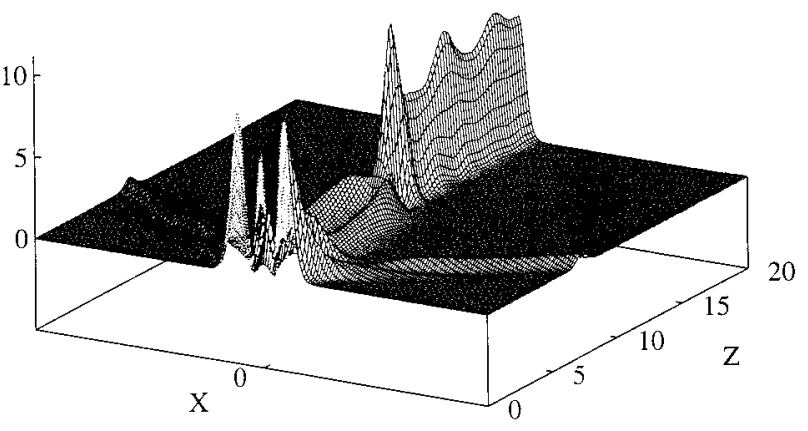

FIG. 5. The evolution of the ordinary fundamental (input as $\mathrm{TEM}_{20}$ ) intensity in the $x-z$ plane for $I_{0}=15 \mathrm{GW} / \mathrm{cm}^{2}$ without walk off. The extraordinary input is $\mathrm{TEM}_{00}$.

waves and solitons predicted by the simple model. ${ }^{4}$ Figure 5 shows the evolution of the beams for $\mathrm{TEM}_{20}$ (three maxima along the $x$ axis) ordinary and $\mathrm{TEM}_{00}$ extraordinary fundamental beams. For $I_{0} \sim 15 \mathrm{GW} / \mathrm{cm}^{2}$, the propagation directions of the three middle beams are very close so that they first merge to form two solitary waves which subsequently merge to form a single soliton. Such beam fusion depends on many factors; the beam propagation directions, the relative phases of the two fundamentals, and the walk off. Note that the peak intensity of the output central beam in Fig. 5 is much higher than expected from the zone model. It could result in anomalously low measurements of damage thresholds performed with multimode lasers.

In conclusion, we have demonstrated the formation of multiple 2D spatial solitons in a quadratic medium when excited with different laser modes of Type II SHG. In general, for identical $\mathrm{TEM}_{m n}$ inputs, up to $(m+1) \times(n+1)$ spatial solitons can be generated. For different input laser modes, a simple, beam overlap, zone model was successful in predicting the maximum number of solitons generated. Although beam walk off was found to change the threshold intensities for emission of various solitons, the general features were the same. Soliton fusion, especially for higher order modes, was found to reduce the maximum number of solitons and lead to very high peak intensities.

This research was supported by ARPA, ARO, and AFOSR.

${ }^{1}$ For example, S. A. Akhmanov, R. V. Khokhlov, and A. P. Sukhorukov, in Laser Handbook, edited by F. T. Arecchi and E. O. Schultz-DuBois (North Holland, Amsterdam, 1972), pp. 1151-1228.

${ }^{2}$ Y. N. Karamzin and A. P. Sukhorukov, JETP Lett. 20, 339 (1974); Zh. Eksp. Teor. Fiz. 68, 834 (1975) [Sov. Phys. JETP 41, 414 (1976)].

${ }^{3}$ W. E. Torruellas, Z. Wang, D. J. Hagan, E. W. VanStryland, G. I. Stegeman, L. Torner, and C. R. Menyuk, Phys. Rev. Lett. 74, 5036 (1995).

${ }^{4}$ D.-M. Baboiu, G. I. Stegeman, and L. Torner, Opt. Lett. 20, 2282 (1995). 